\title{
La réhabilitation du sourire gingival en orthodontie
}

\section{RÉSUMÉ}

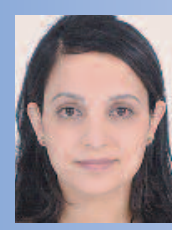

Asmae BENKADDOUR Résidente au département d'orthopédie dento-faciale, Faculté de médecine dentaire de Rabat, Université Mohammed V - Souissi, Avenue Allal Al Fassi, rue Mohamed Jazouli, Campus universitaire Al Irfane, B.P. 6212 Rabat Instituts, Rabat, Maroc.

\section{Wiam RERHRHAYE}

Professeur agrégé au département d'orthopédie dento-faciale, Faculté de médecine dentaire de Rabat, Université Mohammed V - Souissi.

\section{Zouhair ISMAILI}

Professeur agrégé,

Chef du département de parodontologie, Faculté de médecine dentaire de Rabat, Université Mohammed V - Souissi.

\section{Mohammed Faouzi AZAROUAL} Professeur agrégé

au département d'orthopédie dento-faciale, Faculté de médecine dentaire de Rabat, Université Mohammed V - Souissi.

\section{Salwa REGRAGUI}

Professeur agrégé,

Chef du département

d'orthopédie dento-faciale,

Faculté de médecine dentaire de Rabat,

Université Mohammed V - Souissi.

\section{Elhoussine AALLOULA}

Professeur de l'enseignement supérieur, Chef de service d'orthopédie dento-faciale, Faculté de médecine dentaire de Rabat, Université Mohammed V - Souissi.
L'esthétique constitue un véritable thème d'actualité et, parmi ses critères, le sourire occupe une place de plus en plus importante. II constitue une partie essentielle de l'interaction de l'individu avec son environnement.

Un sourire brillant, équilibré et harmonieux représente la demande esthétique de nos patients.

Le sourire gingival quant à lui est caractérisé par l'exposition d'une quantité importante de gencive au cours du sourire. Il est donc considéré comme disgracieux et affectant l'esthétique et par conséquent la communication non verbale.

Notre travail va porter sur le traitement multidisciplinaire du sourire gingival incluant orthopédie, orthodontie, chirurgie orthognathique, chirurgie parodontale ainsi qu'un traitement par injection de toxine botulique type $\mathrm{A}$. 


\section{Introduction}

$>$

Le sourire occupe une place privilégiée dans I'esthétique du visage, il joue un rôle déterminant aussi bien dans l'expression et I'apparence faciale que dans l'interaction sociale de l'individu.

Un sourire équilibré, uniformisé, dento-labial, avec un contour gingival harmonieux, des dents blanches, bien alignées, encadrées par des lèvres aux proportions harmonieuses, constitue la demande esthétique principale de nos patients (fig. 1).

Le sourire gingival n'entre pas dans ces critères et est donc considéré comme étant disgracieux et affectant l'esthétique et la communication non verbale.

Dans ce travail, nous proposons de passer en revue les différentes étiologies du sourire gingival, ainsi que le traitement multidisciplinaire incluant orthopédie, orthodontie, chirurgie orthognathique, chirurgie parodontale et traitement par injection de toxine botulique type A.

Le sourire est dit gingival si plus de $3 \mathrm{~mm}$ de gencive sont visibles pendant un sourire retenu [1]. D'après l'étude de Hunt et Johnson en 2002, l'exposition gingivale durant le sourire est considérée comme inesthétique. L'indice de beauté est attribué aux sourires dont l'exposition gingivale maximale est comprise entre 0 et $2 \mathrm{~mm}$; avec idéalement un sourire n'exposant pas de gencive. Cependant l'indice d'attraction diminue considérablement avec $3 \mathrm{~mm}$ ou plus d'exposition gingivale [2].

L'étude de Geron et Atalia a rapporté qu'une exposition gingivale de $1 \mathrm{~mm}$ lors du sourire est jugée inesthétique par le public. Le sourire devient moins attrayant au fur et à mesure que la quantité de gencive exposée augmente [3].

\section{Étiologies du sourire gingival}

Le sourire gingival peut être dû à une malformation du squelette qui se caractérise par un excès du maxillaire supérieur dans le plan vertical [4], ou à une anomalie dento-alvéolaire (supraclusion antérieure). L'éruption passive incomplète et l'accroissement gingival sont aussi incriminés dans l'étiologie de l'exposition gingivale excessive $[5,6]$.

Le sourire gingival peut être aussi causé par une lèvre supérieure trop courte avec participation de la forme du nez [7], ou par I'hyperactivité des muscles releveurs de la lèvre supérieure [8]. Il est également important d'évaluer la longueur de la couronne clinique, car une cou- ronne trop courte peut causer l'exposition excessive des gencives [5].

Étiologie basale :

l'excès maxillaire antérieur

Lorsque le patient expose une large portion de gencive au sourire (> $3 \mathrm{~mm}$ ), le diagnostic du syndrome « face longue » doit être évoqué (fig. 2). Le diagnostic de l'excès vertical antérieur dépend de la combinaison de trois principales caractéristiques:

- augmentation de l'angle mandibulaire (angle goniaque ouvert) ; 

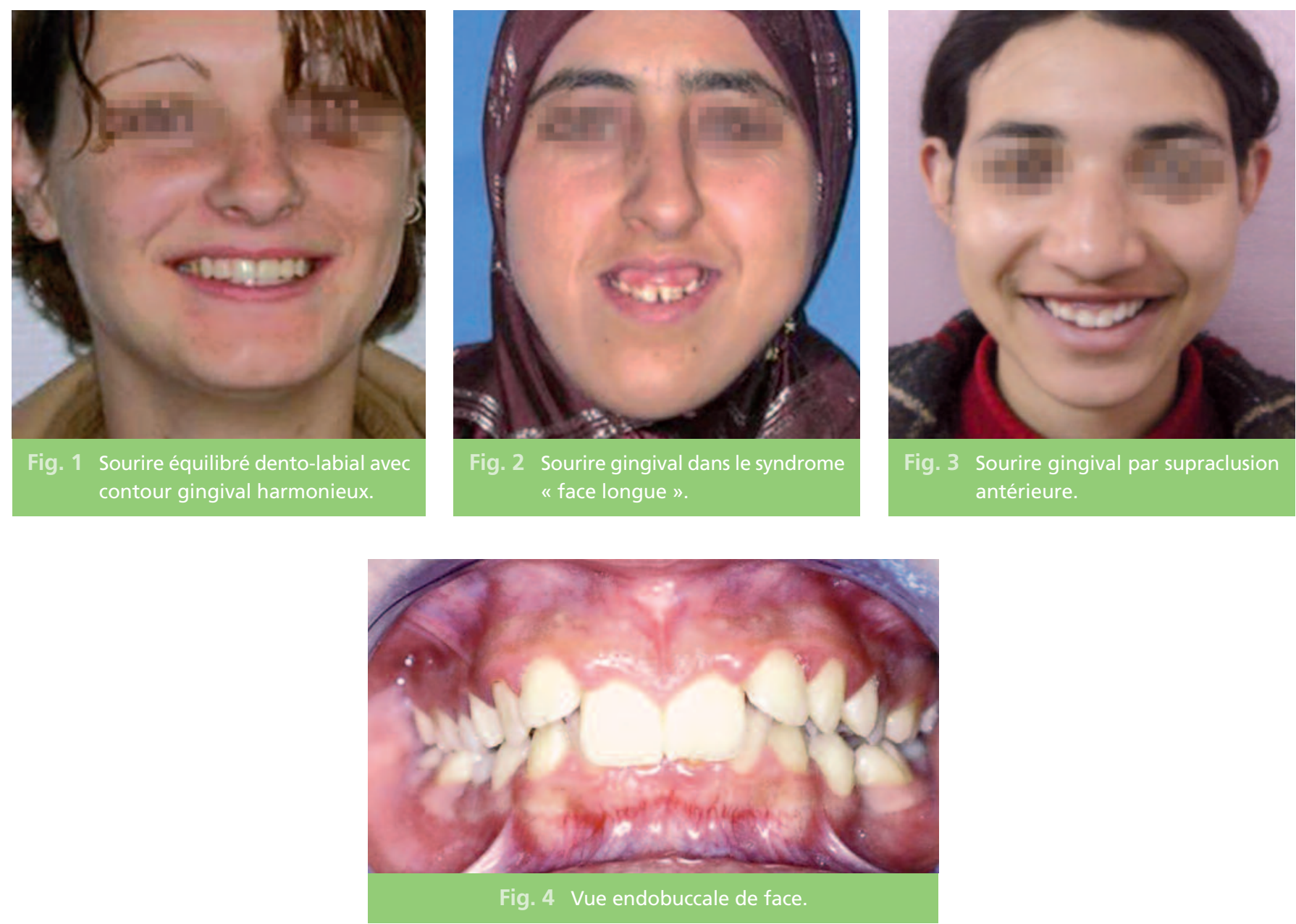

- augmentation de la hauteur totale de la face ;

- diminution de l'index facial [7].

\section{Étiologie dento-alvéolaire} (supraclusion antérieure)

La supraclusion antérieure est une anomalie dento-alvéolaire du sens vertical, localisée au secteur alvéolaire antérieur et caractérisée par un recouvrement incisif excessif qui dépasse $3 \mathrm{~mm}$ (fig. 3). Cette anomalie est le plus souvent symétrique, uni- ou bimaxillaire.

Quand elle est d'origine maxillaire, elle peut se limiter aux deux incisives centrales qui sont plus basses que le plan d'occlusion maxillaire, ou peut intéresser les quatre incisives ou le secteur incisivo-canin. Le bord libre des incisives supérieures est parfois trop bas par rapport au stomion [9]. La supraclusion est une compensation verticale dento-alvéolaire, liée à l'éruption excessive des incisives supérieures. L'os alvéolaire et la gencive migrent vers le bas accompagnant les dents, ce qui a pour conséquence l'apparition d'un sourire gingival disgracieux [6] (fig. 4).

\section{Éruption passive incomplète}

L'éruption passive incomplète se manifeste cliniquement par le recouvrement d'une partie des couronnes dentaires par la gencive. 
L'éruption passive altérée est classée en deux types distincts (fig. 5) :

- type $I$ : on retrouve une quantité importante de gencive (> à $5 \mathrm{~mm}$ ) lorsqu'on la mesure de la gencive marginale libre jusqu'à la ligne mucco-gingivale.

Le type I est subdivisé en deux sous catégories : la sous-catégorie IA, la distance entre le niveau de la jonction amélo-cémentaire et la crête osseuse est $>1 \mathrm{~mm}$; la sous-catégorie IB, la distance entre la jonction amélo-cémentaire et la crête osseuse est $<1 \mathrm{~mm}$;

- type II : la dimension de la gencive mesurée de la gencive marginale libre à la ligne muccogingivale est normale (3 à $5 \mathrm{~mm}$ ).

Cette classification prend toute son importance dans le choix de la procédure thérapeutique $[5,6]$.

\section{Accroissement gingival}

L'accroissement gingival constitue un véritable préjudice esthétique pour le patient qui dévoile un sourire gingival disgracieux [5].
C'est une augmentation de volume, située au niveau des papilles interdentaires et de la gencive marginale. II regroupe à la fois I'hyperplasie et l'hypertrophie gingivale [10].

\section{Étiologie labiale}

\section{> Hyperfonction musculaire}

Une autre cause du sourire gingival est la capacité musculaire à soulever la lèvre supérieure plus haut que la moyenne (fig. 6).

Si l'analyse céphalométrique du maxillaire montre la présence de dimensions normales, et si le patient expose $2 \mathrm{~mm}$ de la hauteur des incisives au repos et une grande quantité de gencive au sourire, le sourire gingival peut être le résultat de l'hyperfonction des muscles élévateurs de la lèvre supérieure [8].

\section{> Longueur des lèvres}

La longueur des lèvres présente aussi une grande influence sur la quantité de dents exposées au repos et au sourire. Une lèvre supérieure courte constitue une cause fréquente du sourire gingival [7] (fig. 7).

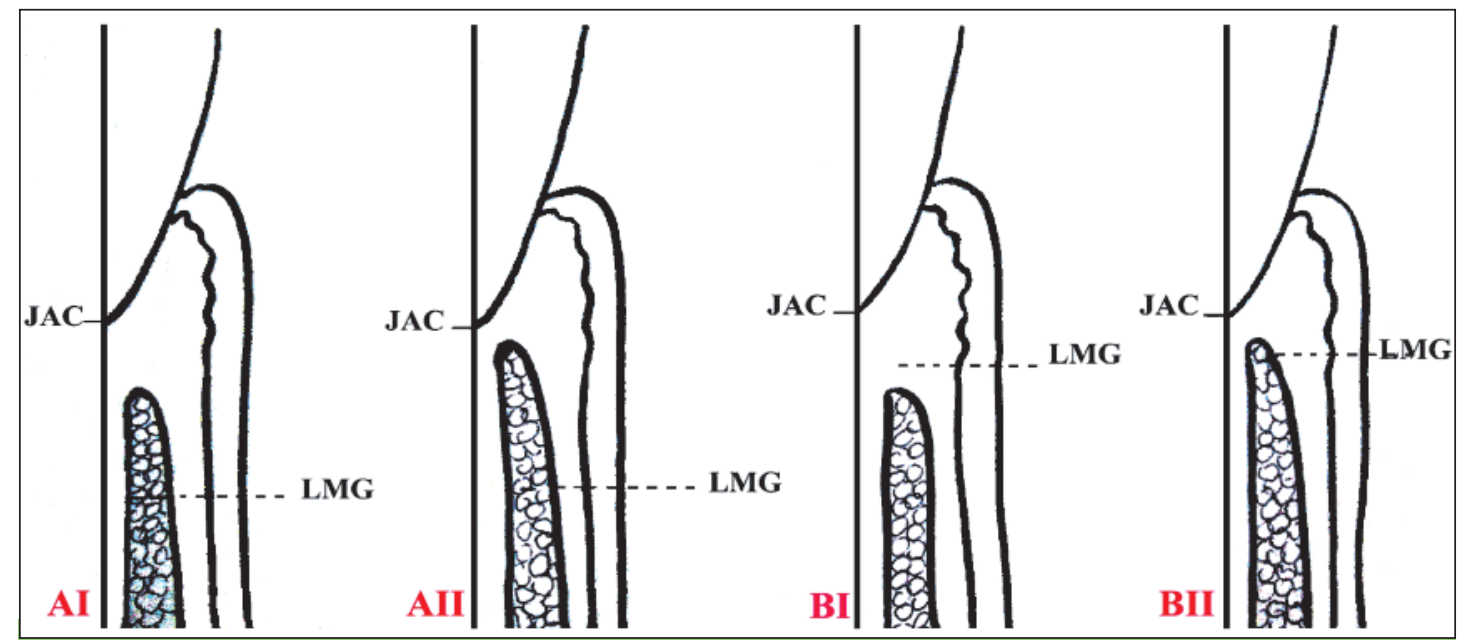

Fig. 5 Les types d'éruption passive incomplète (JAC : jonction amélo-cémentaire ; LMG : ligne mucco-gingivale). 
La lèvre supérieure a été mesurée au repos, de la racine du nez jusqu'au bord inférieur de la

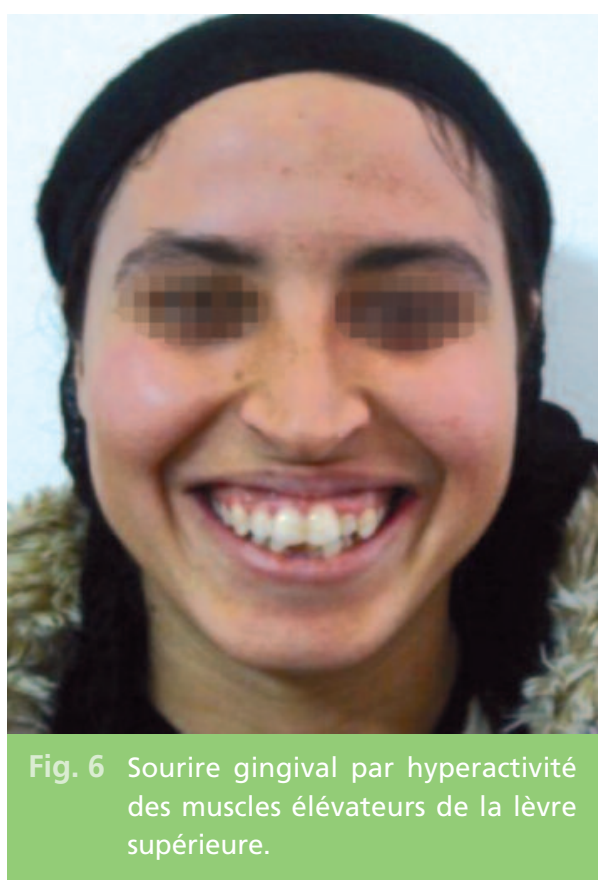

lèvre supérieure, sa longueur moyenne varie entre 19 et $22 \mathrm{~mm}$ [11].

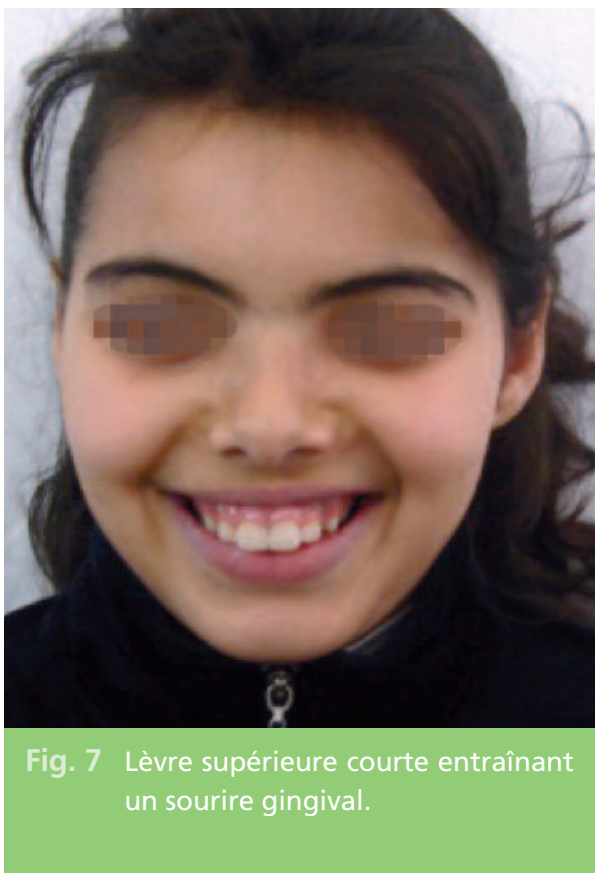

\section{Moyens de réhabilitation du sourire gingival}

Un sourire attrayant, bien équilibré, est l'objectif suprême de la thérapeutique orthodontique moderne. Le jugement esthétique doit être fait en regardant le patient dans divers états statique et dynamique.

Le sourire gingival compte parmi les anomalies du sourire constituant le souci des patients et des cliniciens, qui le trouvent esthétiquement indésirable.

La solution thérapeutique inclut diverses combinaisons : orthopédique, orthodontique, chirurgicale et parodontale selon le diagnostic établi. À côté de ces moyens, un traitement par toxine botulique peut être envisagé.

\section{Moyens interceptifs}

La plaque interincisive représente la base du traitement interceptif, c'est une plaque en résine qui s'oppose par sa partie antérieure à l'égression des incisives maxillaires [12].

On peut également utiliser un activateur classique, auquel on ajoute un prolongement recouvrant la zone d'éruption des incisives, il aura le même rôle que la plaque interincisive. De même, si la promaxillie est traitée par une force extra-buccale montée sur une gouttière qui englobe toute l'arcade maxillaire, la gouttière peut alors empêcher l'égression des inci- 
sives supérieures en s'adaptant à la gencive de la zone d'éruption [12].

Ces moyens concernent en particulier le jeune enfant, avant éruption des incisives supérieures permanentes, et ils ont pour but d'empêcher le développement d'une supraclusion qui doit être diagnostiquée dès la denture lactéale.

\section{Traitement orthodontique fixe}

L'objectif du traitement orthodontique est d'obtenir l'ingression des incisives maxillaires. Cette ingression peut s'accomplir selon différentes techniques.

\section{$>$ Forces directionnelles \\ à traction haute sur un arc continu au maxillaire}

Ce système se compose d'un appui crânien et de barrettes de Wittmann, qui s'accrochent en avant à l'arc Edgewise et sont tirées en arrière par un système élastique ancré sur l'appui crânien (fig. 8 a et b).

Il est surtout indiqué en cas de sourire gingival par supraclusion incisive maxillaire. II permet le contrôle antérieur du plan d'occlusion voire même la bascule anti-horaire de celui-ci sans entraîner l'égression d'une molaire ou d'une autre dent. II permet l'ingression simultanée des incisives et des canines [12].

\section{$>$ Mini-implants}

La correction du sourire gingival peut se faire aussi par I'utilisation de mini-vis (fig. 9). Cet ancrage peut fournir une véritable ingression des incisives maxillaires sans provoquer d'extrusion molaire. Les mini-vis peuvent être insérées au niveau de l'os alvéolaire vestibulaire entre les incisives centrales, et en dessous de l'épine nasale antérieure pour permettre l'in-
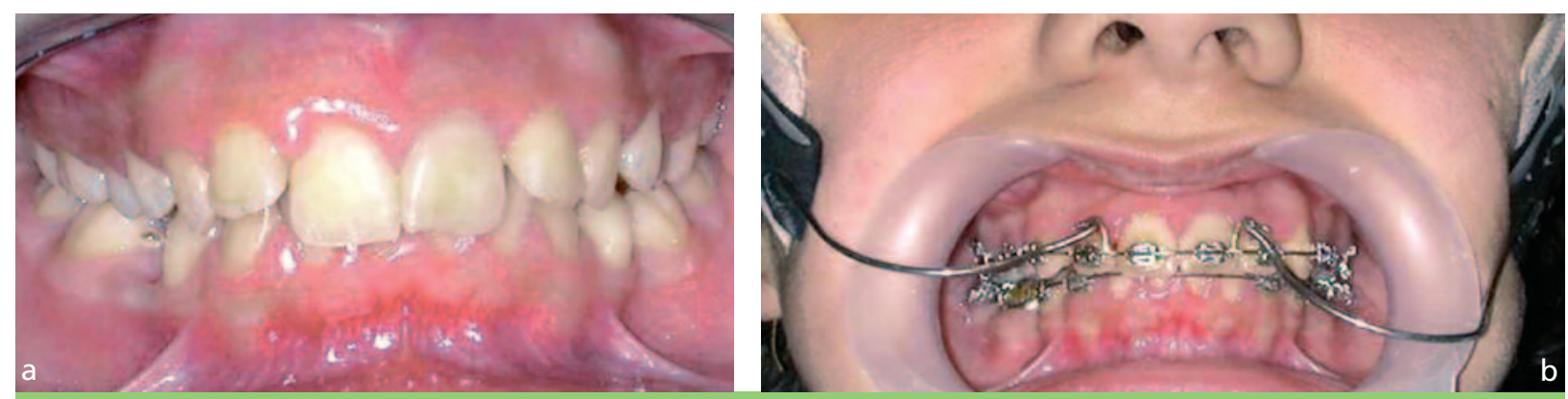

Fig. 8 a et b Correction du sourire gingival par forces directionnelles.
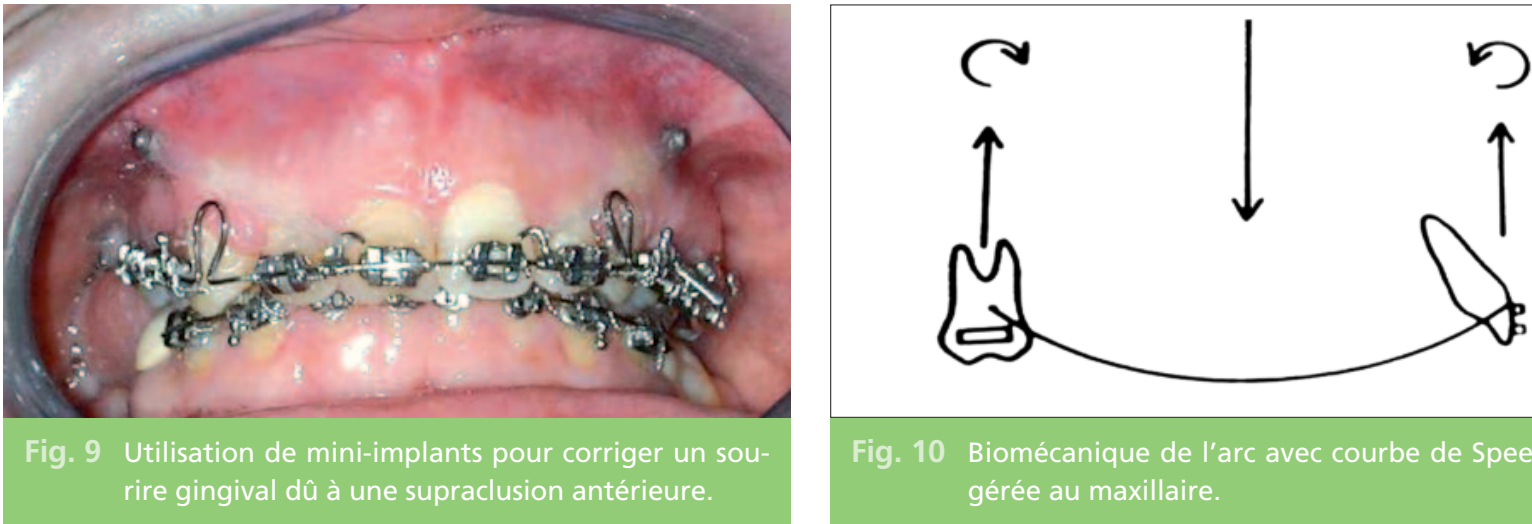

Fig. 10 Biomécanique de I'arc avec courbe de Spee exagérée au maxillaire. 
gression des deux incisives centrales dans un premier temps, puis l'ingression des quatre incisives dans un deuxième temps. Les incisives maxillaires seront ingressées et basculées légèrement en direction vestibulaire sans mobilisation des premières molaires.

Si une proalvéolie supérieure existe, les mini-vis sont placées en distal des canines, ou entre canines et incisives latérales pour permettre ingression et recul simultanés [13].

\section{> Technique segmentée: système de Ricketts et de Burstone}

D'autres techniques sont utilisées pour l'ingression incisive : le système de Ricketts [12] et le système de Burstone $[12,14]$ sont des dispositifs à forces légères, qui permettent l'ingression incisive en s'appuyant sur les molaires.

\section{$>$ Arc continu avec une courbe de Spee exagérée au maxillaire}

Un arc continu avec une courbe de Spee exagérée au maxillaire est conçu pour réduire la supraclusion.

Cet arc entraîne, dans un premier temps, une ingression molaire avec une légère version distale et vestibulaire, une égression prémolaire, ce qui augmente la dimension verticale et diminue la supraclusion et une vestibulo-version incisive (fig. 10).

Mais les prémolaires égressées ne peuvent à elles seules tenir la dimension verticale; elles se ré-ingressent dans un deuxième temps et c'est au cours de cette ré-ingression et sous l'influence des muscles élévateurs que s'ingressent les incisives [12] (fig. 11).
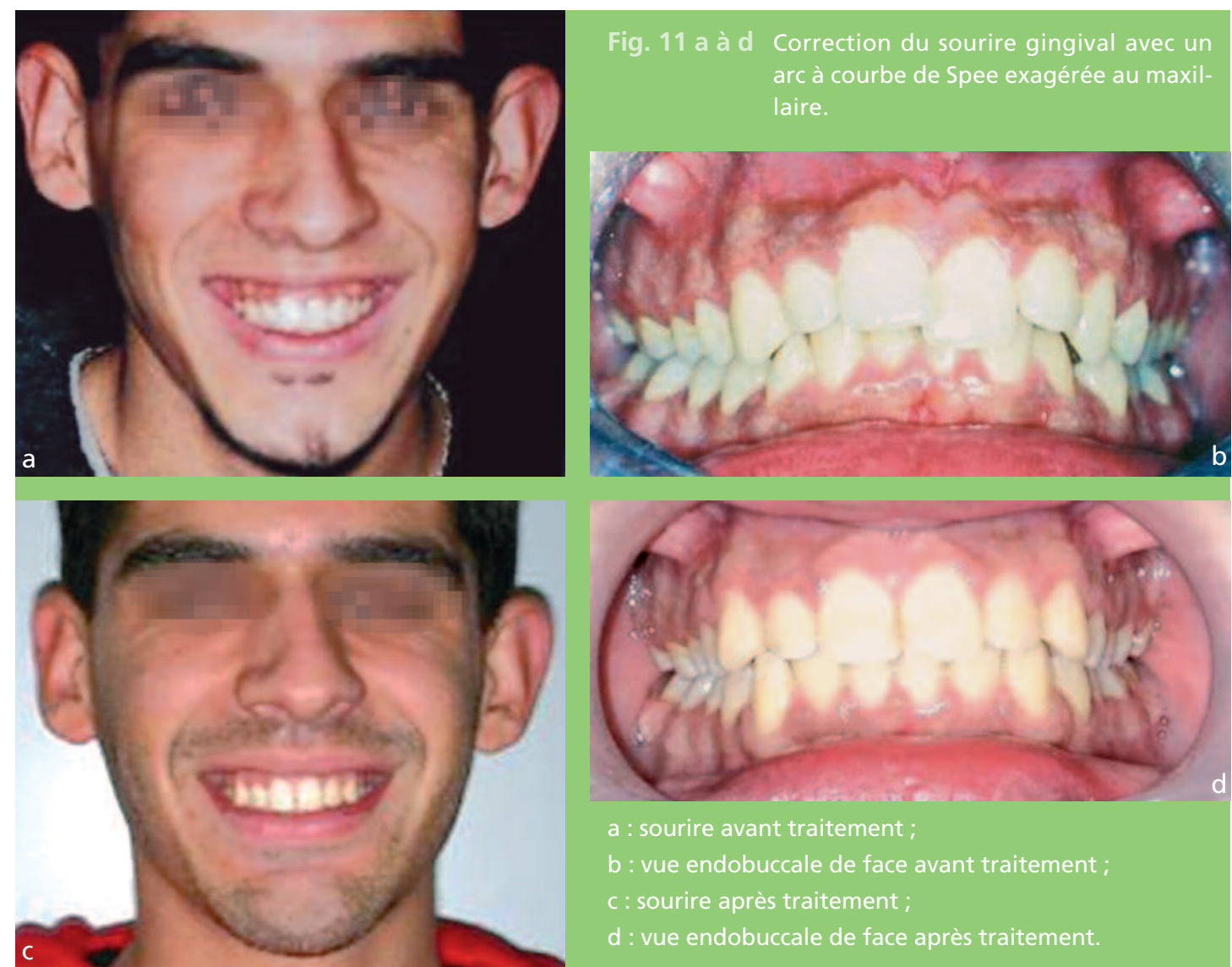


\section{Technique linguale}

La technique linguale à attaches multiples a été aussi décrite par Altounian [15] comme moyen de correction de la supraclusion incisive. Les brackets collés sur les faces linguales des incisives comportent une petite surface plane dite " plan de morsure ", destinée à recevoir le choc occlusal des dents antagonistes. La pression occlusale exercée sur les incisives maxillaires tend à les ingresser. En même temps, les molaires s'égressent $[12,16]$.

Les attaches linguales gênent la prononciation pendant quelques jours, I'alimentation pendant quelques semaines. Les décollements sont peu fréquents car la force occlusale est vite modérée par un réflexe proprioceptif.

Les résultats de la méthode ont été précisément étudiés par Altounian et al., il a été conclu que [12] :

- les incisives mandibulaires s'ingressent beaucoup plus que les maxillaires ;

- les molaires maxillaires restent stables alors que les mandibulaires s'égressent légèrement.

\section{Traitement chirurgical}

\section{$>$ Repositionnement supérieur du maxillaire (ostéotomie Lefort I)}

Le repositionnement supérieur du maxillaire est la méthode de choix pour corriger l'excès vertical du maxillaire entraînant un sourire gingival (fig. 12).

La technique chirurgicale consiste à impacter le maxillaire antérieurement par l'ostéotomie de Lefort I. Une préparation orthodontique préchirurgicale peut s'avérer nécessaire afin d'aligner les arcades, de diminuer des compensations alvéolaires si elles existent et de coordonner l'arcade maxillaire à l'arcade mandibulaire dans les trois sens de l'espace $[17,18]$.

\section{$>$ Ostéotomie segmentaire de Wassmund}

L'ostéotomie segmentaire de Wassmund peut être utilisée dans la correction des supraclusions incisives. L'intervention est limitée au secteur alvéolaire antérieur, la correction est obtenue par une bascule postérieure et une élévation du bloc réséqué.

Son indication principale est la supraclusion par supra-alvéolie incisive supérieure [19].

\section{$>$ Chéiloplastie V-Y}

En présence d'un excès vertical du maxillaire antérieur, associé à un philtrum court responsable du sourire gingival, l'augmentation de la longueur de la lèvre supérieure peut être réalisée par une chéiloplastie en $\mathrm{V}$-Y en même temps que la chirurgie de LeFort I (fig. 13) [7].

\section{Chirurgie parodontale}

Les moyens permettant la correction du sourire gingival en chirurgie parodontale sont : la gingivectomie et le lambeau repositionné apicalement. Cette chirurgie peut être effectuée seule ou associée à un traitement orthodontique.

La gingivectomie est indiquée en cas d'éruption passive incomplète et en cas d'accroissement gingival.

Dans le cas d'éruption passive incomplète de type IA, la réalisation d'une gingivectomie à biseau interne est suffisante pour le traitement. Une résection osseuse associée à un remodelage gingival est nécessaire en cas d'éruption passive incomplète de type IB.

Dans le type II, il n'y a pas d'excès de gencive, le recours à un lambeau déplacé apicalement avec ou sans résection osseuse est nécessaire ; la réalisation d'une gingivectomie n'est pas indiquée $[4,5]$ (fig. 14). 

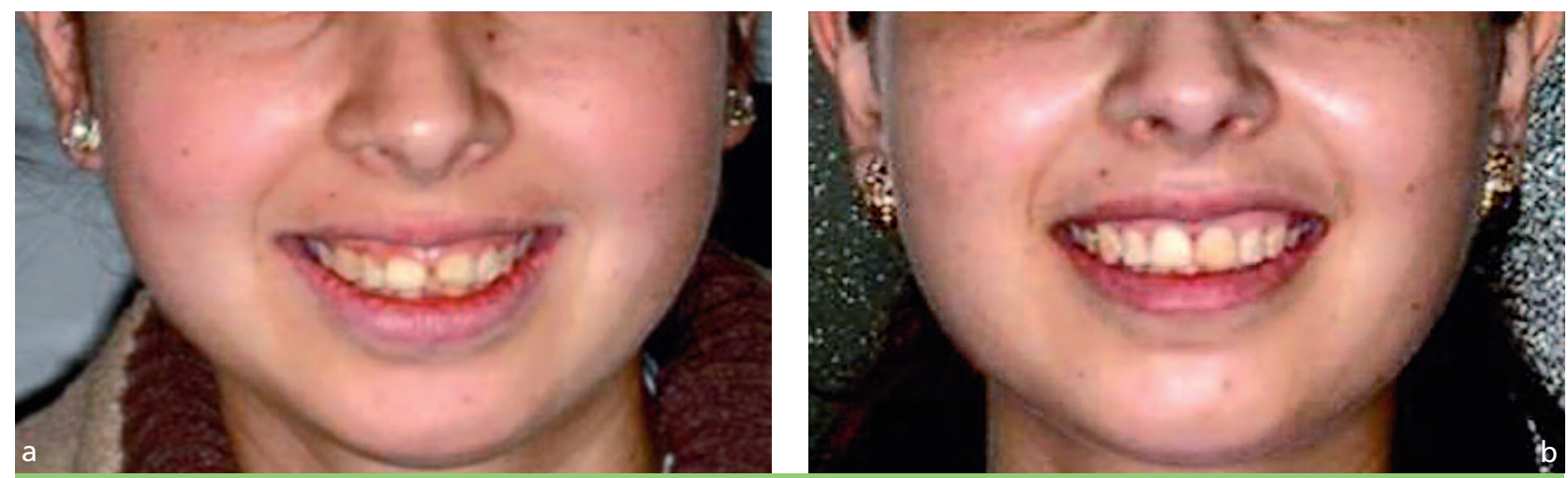

Fig. 12 a et b Sourire avant et après traitement chez la même patiente.
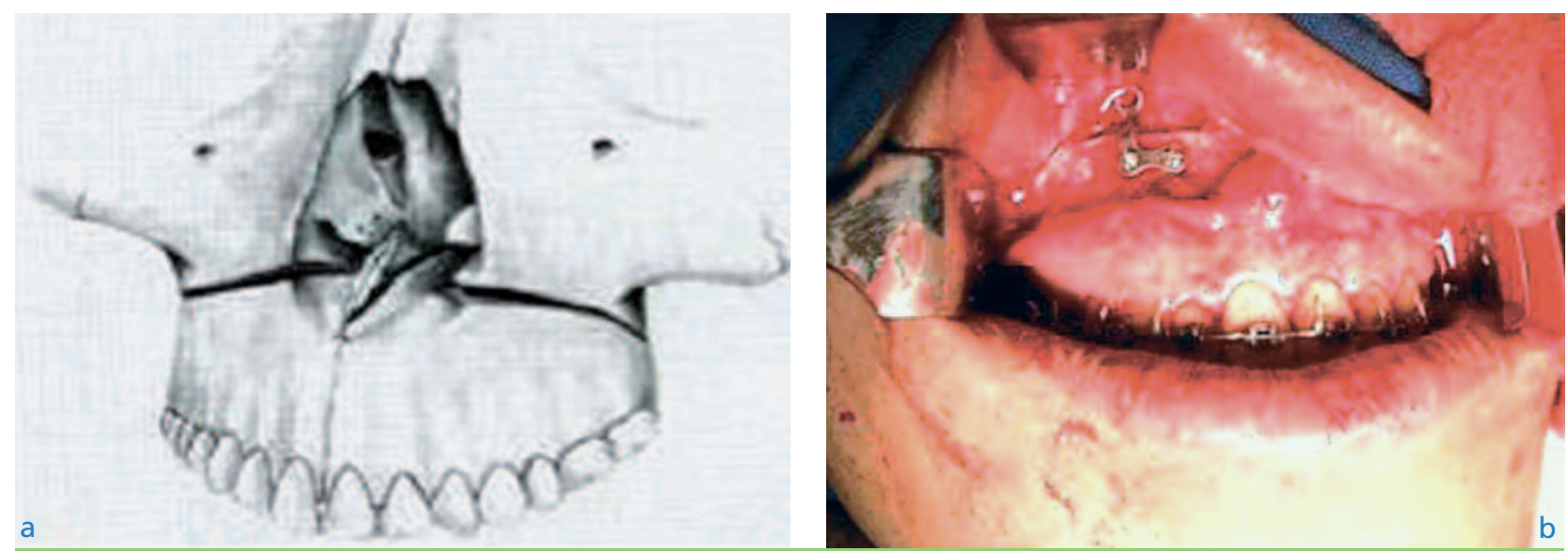

Fig. 13 a et b Chirurgie Lefort I d'impaction maxillaire.
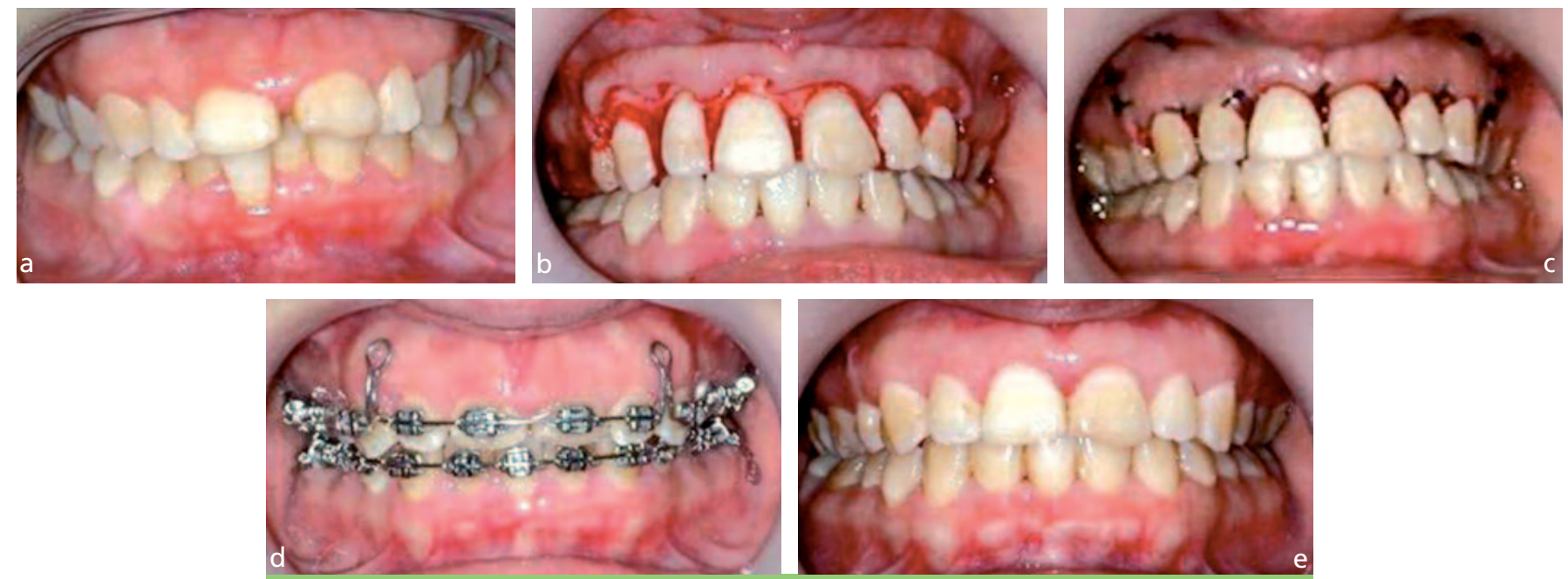

Fig. 14 a à e Réhabilitation du sourire gingival par orthodontie associée à un lambeau positionné apicalement. 


\section{Toxine botulique}

L'injection de toxine botulique est une procédure efficace, légèrement invasive, qui améliore temporairement le sourire gingival en cas d'hyperactivité musculaire.

\section{Conclusion}

Actuellement, le sourire gingival est perçu par le public et les praticiens comme étant disgracieux et affectant l'esthétique du sourire, surtout quand il dépasse $3 \mathrm{~mm}$. Son étiologie peut associer un ou plusieurs facteurs, et son traitement intègre plusieurs disciplines selon
D'après l'étude de Manio-Polo, l'effet de l'injection commence à se faire sentir à partir du dixième jour avec un résultat optimal au bout de 14 jours. Ces résultats sont réversibles au bout de 3 à 6 mois, d'où la nécessité d'injections supplémentaires [8].

I'étiologie et la sévérité de l'exposition gingivale.

Nous pouvons donc agir considérablement sur l'image et la beauté du sourire de nos patients, qui sont devenus de plus en plus demandeurs en matière d'esthétique dento-faciale.

\section{Bibliographie}

1. Allen EP.

Use of mucogingival procedures

to enhance esthetics. Dent Clin North Am 1988;32: 307-30.

2. Hunt $O$, Johnson $C$, Hepper P, Burden D, Stevenson $\mathrm{M}$.

The influence of maxillary gingival exposure on dental attractiveness rating. Eur J Orthod 2002;24:199-204.

3. Geron S, Atalia W. Influence of sex on the perception of oral and smile esthetics with different gingival display and incisal plane inclination. Angle Orthod 2005;75(5):778-784.
4. Ezquerra FMD, Berrazueta M. New approach to the gummy smile. Plast Reconstr Surg 1999;104(4):1143-50.

5. Borghetti A, Monnet-Corti V. Chirurgie plastique parodontale. Collection JPIO. Paris : Éditions CDP, 2000.

6. Foley $\mathrm{T}$, Sandhu $\mathrm{H}$, Athanasopoulos $\mathrm{C}$. Esthetic periodontal considerations in orthodontic treatment. The management of excessive gingival display. J Can Dent Assoc 2003;69(6):368-72.

7. Sarver DM, Rousso DR. Plastic surgery combined with orthodontic and orthognatic procedures.
Am J Orthod Dentofacial Orthop 2004;126(3):305-7.

8. Polo $\mathrm{M}$.

Botulinum toxin type A

in the treatment of excessive gingival display. Am J Orthod Dentofacial Orthop 2005;127(2):214-8.

9. Bassigny $\mathrm{F}$. Manuel d'orthopédie dento-faciale. Paris : Masson, 1983.

10. Harel-Raviv M, Eckler M, Lalani K, Raviv E, Gornitsky M. Nifedipine-induced gingival hyperplasia.

A comprehensive review and analysis. Oral Surg Oral Med Oral Pathol Oral Radiol Endod 1995:79(6):715-22. 
11. Peck S, Peck L, Kataja M. The gingival smile line.

Angle Orthod 1992;62(2):91-100.

12. Phillippe J.

La supraclusion et ses traitements. Paris : Éditions SID, 1995.

13. Kim TW, Kim H, Lee SJ. Correction of deep overbite and gummy smile by using a mini-implant with a segmented wire in a growing Classe II Division 2 patient. Am J Orthod Dentofacial Orthop 2006;130(5):676-85.
14. Deblock L, Ray B, Guingamp N, Petitpas L. Biomécanique de l'ingression incisive : son contrôle en technique segmentée de Burstone. Rev Orthop Dento Faciale 1997;31(2):183-98.

15. Altounian G.

Les attaches linguales à insertion horizontale. In: Philippe J. L'orthodontie de l'adulte.

Paris : Éditions SID, 1989:115-80.

16. Sébastien N, Guillaume J. Orthodontie linguale : le choix du système.
Rev Orthop Dento Faciale 2007;41:9-24.

17. Angelillo JC, Dolan EA. The chirurgical correction of vertical maxillary excès. Ann Plast Surg 1982;8(1):64-70.

18. Kawamato HK. Treatment of the elongated lower face and gummy smile.

Clin Plast Surg 1982;9:479-89.

19. Garcia R.

Le point sur les protocoles chirurgico-orthodontiques. Actual Odonto Stomatol 1994;48(187):447-76.

\section{SUMMARY}

\section{Reabilitation of the gummy smile in orthodontics}

Asmae BENKADDOUR,

Wiam RERHRHAYE,

Zouhair ISMAILI,

Mohammed Faouzi AZAROUAL, Salwa REGRAGUI,

Elhoussine AALLOULA

\section{Keywords \\ - sourire gingival \\ - étiologie \\ traitement}

The aesthetic makes a real theme of the current affairs, and among its elements, the smile occupies an increasingly important place. It constitutes an essential part of the social interaction of the individual.

A shining, balanced and harmonious smile constitutes the aesthetic request of our patients.

The gummy smile is characterized by the exposure of an important quantity of gum during the smile. And thus, it is considered as being unaesthetic, disgraceful, and affecting non verbal communication.

Our work concerns especially the multidisciplinary therapeutic of gummy smile witch includes orthodontics and orthopedics, orthognathic surgery, periodontal surgery and treatment by injection of Botulinum toxin type $A$ 